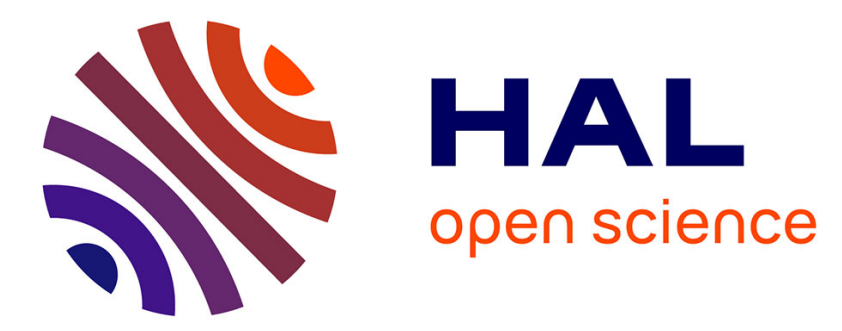

\title{
Multi-criteria Performance Assessment of Adaptive Radar Resources Management: Application to Naval Scenario
}

Christophe Labreuche, Cédric Buron, Peter W Moo, Frédéric Barbaresco

\section{To cite this version:}

Christophe Labreuche, Cédric Buron, Peter W Moo, Frédéric Barbaresco. Multi-criteria Performance Assessment of Adaptive Radar Resources Management: Application to Naval Scenario. The 20th International Radar Symposium (IRS), Jun 2019, Ülm, Germany. hal-02090506

\author{
HAL Id: hal-02090506 \\ https://hal.science/hal-02090506
}

Submitted on 17 Apr 2019

HAL is a multi-disciplinary open access archive for the deposit and dissemination of scientific research documents, whether they are published or not. The documents may come from teaching and research institutions in France or abroad, or from public or private research centers.
L'archive ouverte pluridisciplinaire HAL, est destinée au dépôt et à la diffusion de documents scientifiques de niveau recherche, publiés ou non, émanant des établissements d'enseignement et de recherche français ou étrangers, des laboratoires publics ou privés. 


\title{
Multi-criteria Performance Assessment of Adaptive Radar Resources Management: Application to Naval Scenario
}

\author{
Christophe Labreuche * Cédric L R Buron * Peter ${ }^{*}$ Moo ${ }^{\dagger}$ \\ Frédéric Barbaresco ${ }^{\ddagger}$
}

\begin{abstract}
Multifunction radars (MFR) must achieve their capability requirements in an increasingly complex environment, populated with diverse and hostile targets (e.g. low Radar Cross-Section, low speed targets in clutter or high speed, ballistic targets) in saturating scenarios (due to e.g. RF interference or threats). These radar systems are increasingly exploiting active electronically scanned array (AESA) technology to dynamically schedule the use of multiple functions in a short duration. However, the increasing complexity and adaptive nature of MFR radar makes it very difficult to specify their performance in a way
\end{abstract}

\footnotetext{
*Thales Research \& Technology

1, avenue Augustin Fresnel, 91767 Palaiseau cedex, France

email: \{christophe.labreuche,cedric.buron\}

@ thalesgroup.com

${ }^{\dagger}$ Radar Systems Section, Defence Research and Development Canada

Ottawa, Ontario, Canada email: PETER.MOO@forces.gc.ca

$\ddagger$ Thales Land \& Air Systems, Surface Radar Business Line,

Hameau de Roussigny, F-91470 Limours, France email: frederic.barbaresco@thalesgroup.com
}

which can both deliver the required capability and which can be verified in a cost-effective manner. Due to their multi-function feature, the operational scenarios have a strong impact on MFR radars; their performances should be specified accordingly. Addressing the challenges in this area will benefit via better understood requirements which can be more easily interpreted. After definition of FoM (Figures of Merit) for phased array radar operation and performance, we have computed them on benchmark test scenarios of varying complexity. We describe a new methodology to aggregate these metrics to provide a global notation of MFR radar performances.

Keywords: Multi Criteria Decision Aid Adaptive Radar Resource Management Figures of Merit

\section{Introduction}

A naval multi-function radar conducts multiple functions simultaneously, including tracking, surveillance, fire control, and various other functions. An effective Radar Resource Management (RRM) algorithm will allocate 
the radar's resources efficiently while contributing to a specified mission role (NonCooperative Target Recognition, Kill Assessment,...). For naval radars, RRM has traditionally been conducted in a non-adaptive manner. That is, the control and scheduling of radar tasks is fixed and invariant over time. However, naval vessels are operating in increasingly complex environments. This necessitates the development of adaptive RRM techniques, which vary with the target and interference environment that the vessel is encountering at any given time. RRM techniques have the potential to enhance radar performance compared to non-adaptive techniques, but further study of performance, complexity and robustness needs to be carried out $[21,2,12,1,4,3,23]$.

The contribution of this paper is firstly to improve the general Multi-Criteria Decision Analysis (MCDA) approach for assessing the results of RRM simulations, and secondly to apply the MCDA approach to a naval scenario.

The aim of this work is to evaluate the performance of several candidate RRM algorithms. The efficiency of RRM algorithms shall be measured on multiple metrics covering the main functions of the radar. For instance, the main metric related to tracking is track completeness. As one algorithm is not in generally expected to outperform all other ones on all criteria, one needs MCDA to weigh-up the pros and cons of each solution and to help identifying the most preferred one. We have proposed in previous works the use of a MCDA approach [1,17] supported by a tool called MYRIAD [20], to assess RRM efficiency, which relies on the knowledge of technical experts and/or operational people.

In MCDA, the alternatives to be assessed are measured on a fixed number of metrics; the value of each metric is normalized and these normalized values are aggregated to produce the overall performance level for each alternative. In standard MCDA, there is one and only one value of each alternative on each metric. This is not the case for the evaluation of RRM, as each candidate algorithm is run on multiple scenarios. As a result, there are many instances of each metric for each algorithm. For instance, there is one instance of metric "track completeness" for each target in each scenario. The standard approach to handle these multiple values consists in computing the average value for each metric, and apply the MCDA model with these average values. We have shown some drawbacks of this approach and advocated an alternative approach [17]. In this new approach, the performance is computed for a metric in two steps: Firstly, each value of each metric is normalized; and secondly, these normalized values are aggregated thanks to an Ordered Weighted Average $(O W A)[24,25]$. This aggregation function allows to put more weights to the worst evaluations - thereby expressing pessimism or risk aversion.

The main contribution of this paper is twofold. Firstly, we improve the approach proposed in [17] by proposing a simple elicitation method such as MACBETH, i.e a method to find the parameters of the aggregation function corresponding most to the DM desiderata. The aggregation of metrics is by nature subjective and shall integrate the preferences of some decision maker regarding the relative importance between criteria and satisfaction threshold. The main difficulty is to elicit the weights of the OWA operator in an interpretable and user-friendly way. Secondly, we apply this general approach to a naval scenario. 
The layout of the paper is as follows. The naval scenario is presented in Section 3 . The results of the evaluation are shown in Section 4.

\section{Multi-Criteria Decision Aid}

The purpose of Multi-Criteria Decision Analysis (MCDA) is to represent and construct the preferences of one or several decision makers (DMs) regarding how to compare or evaluate some alternatives (here RRM algorithms). This can be made on the basis of several decision criteria denoted by $N=\{1, \ldots, n\}$. Most of the time, criteria are conflicting, which means that it is not possible to maximize all of them simultaneously. It is therefore required to make compromises among the criteria - that is that improving a criterion implies that one shall deteriorate on another one. MCDA provides a tool to specify the good compromises from the DMs point of view.

Each criterion $i \in N$ is quantified by an attribute - also called metric or Figure of Merit - represented by the set of its possible values $X_{i}$. The alternatives are characterized by a value on each attribute and can be fully described by elements of $X=X_{1} \times \cdots \times X_{n}$. An alternative $x$ can thus be represented by a vector $\left(x_{1}, \ldots, x_{n}\right) \in X$. The goal of MCDA is to define a numerical representation of the preferences of the DMs, expressed as a function $u: X \rightarrow[0,1]$. This function will be used to assess each alternative (in order to provide the overall level of satisfaction of each alternative) or compare them (in order to identify the best alternative). The scale $[0,1]$ can be interpreted as a degree of satisfaction of the DMs about the alternative, 0 corresponding to a totally unsatisfactory alternative, and 1 corresponding to an optimal one. It is classical to write $u$ in the decomposable way [13]: $u(x)=F\left(u_{1}\left(x_{1}\right), \ldots, u_{n}\left(x_{n}\right)\right)$, for all $x \in X$, where the $u_{i}: X_{i} \rightarrow[0,1]$ are the utility functions (also called value functions) and $F:[0,1]^{n} \rightarrow[0,1]$ is called the aggregation function. The utility functions normalize the metrics and return a level of satisfaction regarding only one metric. The aggregation function takes as input the normalized scores and return the overall score.

For the construction of utility functions, we advocate the use of the MACBETH approach $[7,6]$, that relies on two principles. First, the utility $u_{i}$ shall correspond to an interval scale [14]. Second, for any pair of elements ${ }^{1}$ $\left(x_{i}, y_{i}\right) \in X_{i}^{2}$, the DM is asked to provide an assessment of the difference of satisfaction while going from $x_{i}$ to $y_{i}$ (quantified by $\left.u_{i}\left(x_{i}\right)-u_{i}\left(y_{i}\right)\right)$ on an ordinal scale going from "very small" to "extreme". This scale is given up to an affine transformation. The scale is entirely determined based on two reference elements of $X_{i}$ provided by the DMs. These elements are denoted by $\mathrm{O}_{i}$ and $\mathbb{G}_{i}$ and verify:

$$
\left\{\begin{array}{l}
u_{i}\left(\mathbb{O}_{i}\right)=0 \\
u_{i}\left(\mathbb{G}_{i}\right)=1
\end{array}\right.
$$

i.e. criterion $i$ is not satisfied at all at $\mathrm{O}_{i}$ and completely satisfactory at $\mathbb{G}_{i}$. Once this scaling has been performed, the utility functions are fixed and commensurate. The MACBETH approach can be extended to non-linear aggregation functions, as shown in [19].

Let us now switch to the construction of the aggregation model $H$. The most common aggregation function is the weighted sum. One

\footnotetext{
${ }^{1}$ we write $X_{i}^{2}$ the Cartesian product of $X_{i}$ by itself.
} 
of the major limitations of this function is that it assumes the independence among the criteria, while in most of the actual applications, criteria often interact: they can for instance be complementary, which means that the improvement on one criterion will increase the significance of the improvements on the other one. As an example in radar applications, the search and tracking functions are complementary: a radar will have a good performance if both functions have are good. Interaction can also be used for fairness: in this case, it is seen as preferable to have an uncertain situation where, at 50 - 50 chance, either all individuals are well-served or all of them are illserved, rather than a situation where half of individuals are well-served and half of them are ill-served. One of the interesting extensions of the weighted sum is the Choquet integral [5]. This function constitutes a powerful aggregation function in MCDA, because it can represent nonetheless the previous strategies $[9,11,10]$ but also combine many types of interaction in a versatile way. The Choquet integral can be expressed in its simplest form as [10]:

$$
C(f)=\sum_{i \in N} \phi_{i} f_{i}-\sum_{\{i, j\} \subseteq N} \frac{I_{i j}}{2}\left|f_{i}-f_{j}\right|,
$$

where $f \in[0,1]^{n}$. There are two types of parameters in this expression: the importance degree $\phi_{i}$ of criterion $i$, and $I_{i j}$ the interaction degree between criteria $i$ and $j$ []. Note that the weights of the linear part are the importance coefficients $\phi$; if $I_{i j}>0$, the interaction phenomenon penalizes the overall assessment $C_{\mu}(f)$ according to the difference between $f_{i}$ and $f_{j}$. More precisely, if $f_{i}>f_{j}$, a good evaluation of $f$ on criterion $i$ is penalized to a degree $\frac{I_{i j}}{2}$ by a worse evaluation on criterion $j$. If $I_{i j}<0$, the more $f_{i}$ is different from $f_{j}$, the more the interaction phenomenon increases the overall assessment. More precisely, if $f_{i}<f_{j}$, the bad evaluation of $f$ on criterion $i$ is saved to a degree $\frac{\left|I_{i j}\right|}{2}$ by the better evaluation on criterion $j$. Another asset of the Choquet integral is that the evaluation obtained from this model can be explained to the DMs $[15,16,18]$.

The previously described capabilities are implemented in the multi-criteria decision support tool MYRIAD [20].

\section{Naval scenario}

The problem at stake is to compare two solutions:

"NA+NSR" : the standard legacy NonAdaptive algorithm, with No Special Rate;

"A+STU" : the adaptive algorithm with Special Track Update rates for the most threatening targets. It includes three novel adaptive components: Fuzzy Logic Prioritization, Time Balancing Scheduling (TBS), and Adaptive Update Intervals for Tracking [21].

The operational scenario is related to a Ballistic Missile Defense (BMD) mission, in which the ship contributes to the defense against a ballistic missile threat. The role of the radar is to detect and track the ballistic missile. The scenario is situated in a littoral region with a varied clutter background, including sea, land, and urban clutter - see Fig. 1. In the scenario, targets are both surface and air targets. Surface targets include ships and recreational boats. Air targets consist of ballistic missiles, commercial aircrafts, recreational aircrafts, and 


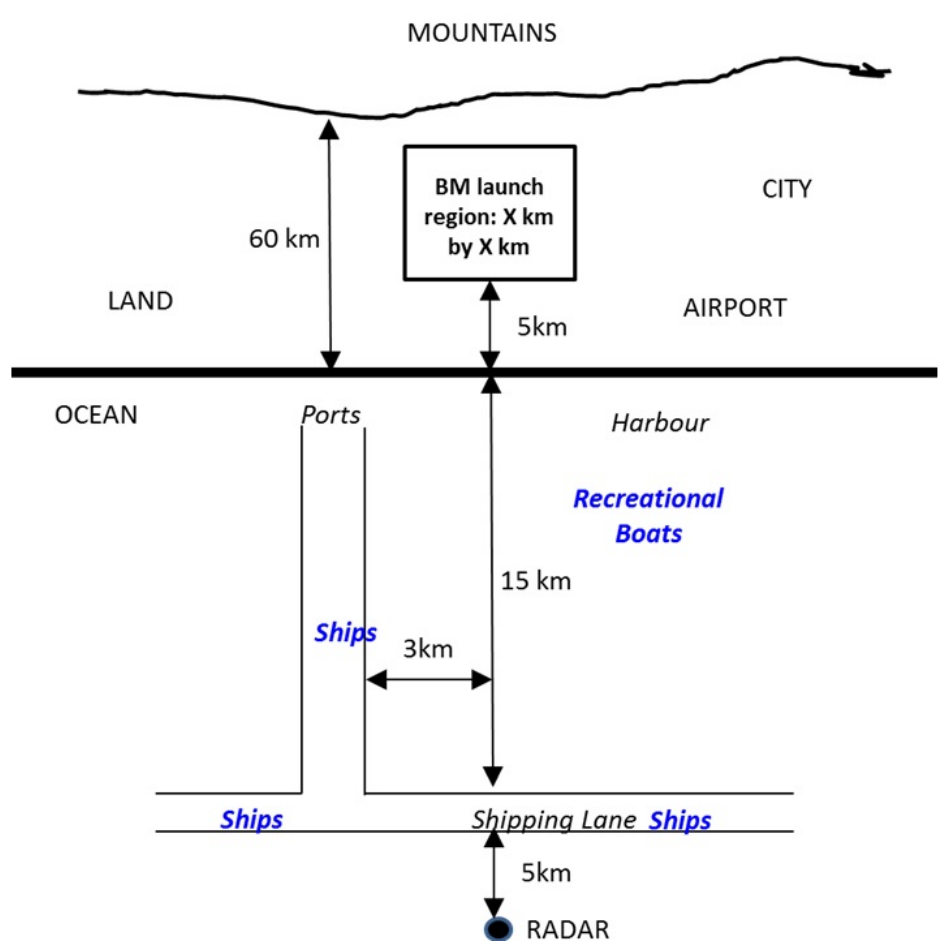

Figure 1: Top down view of a Ballistic Missile (BM) scenario

birds. The ballistic missile launch region is shown as a square region over the land portion of the scenario. As the area of the launch region increases, the radar will require more resources to detect and track a ballistic missile that is launched. The two solutions are evaluated against clutter and non-clutter conditions. Simulation has been performed with Adapt_MFR simulation tool [22].

The evaluation is performed regarding:

- the "Surveillance" viewpoint, measured by metric "Time Frame". This is the average refresh rate of surveillance over the whole space. The smaller the value of this metric, the better;

- the "load balancing" viewpoint, measured by metric "Track Occupancy". This is the percentage of radar time used on tracking - the complementary time being allocated to surveillance. The smaller the value of this metric, the better.

- the "Tracking" viewpoint, measured by metric "Track Completeness". This is the average percentage of each trajectory that is tracked. The larger the value of this metric, the better;

The expectations on track completeness and the severity of non-fulfillment on this metric are not the same for the different targets. Hence, as proposed in [17], we group together the values of track completeness for each type of target - see Fig. 2. 


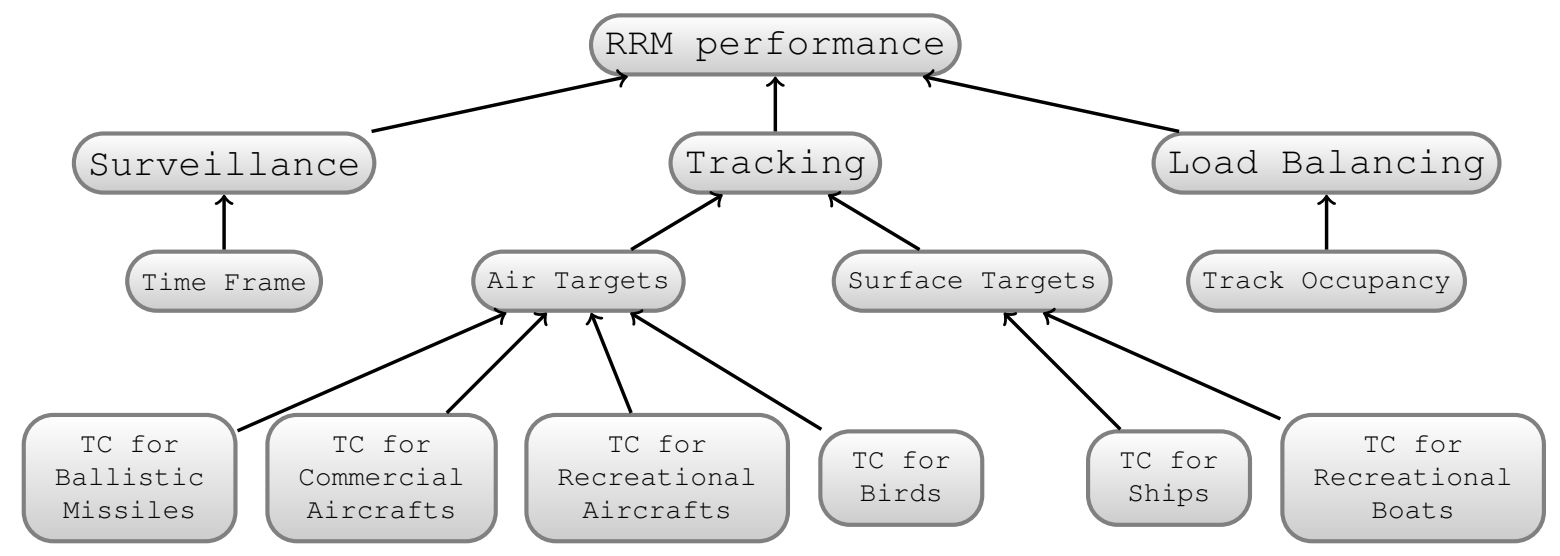

Figure 2: Description of the elementary viewpoints on tracking performance, where TC stands for Track Completeness.

\section{Results}

Due to space limitations, we only present the details of results on tracking function. They are summarized in Fig. 3. They are organized as an array. Columns represent the metrics: "ballistic missile tracking", "commercial aircraft tracking", "recreational aircraft tracking", "bird tracking", "ship tracking" and "recreational boat tracking" from left to right. In the first line, two gauges are represented for each metric: the green ones represent the performance assessment between $0 \%$ (criterion not satisfied at all) and $100 \%$ (criterion perfectly satisfactory) for solution A+STU. The red gauges represent the NA+NSR counterpart.

The following two rows, in green represent the results for solution A+STU. Finally the last two rows represent the counterpart for the $\mathrm{NA}+\mathrm{NSR}$ solution. Among these rows, the third and fifth ones, named "A+STU (metr.) (resp. "NA+NSR (metr.)") show an estimate of the probability distribution over the metric space. These are the raw values of met- ric "track completeness" from 0 (worst value) to 1 (best value) coming from the simulations. The second and fourth raws named "A+STU (crit.) (resp. "NA+NSR (crit.)") show two curves: an estimate of the probability distribution over the the normalized values of the metric (i.e. the utility function applied to the metric), in blue and the corresponding values of the OWA weights, in red. The probabilities have been gotten from the results by the application of a Kernel Density Estimation [8].

Looking at the probability distribution on the metrics, we note that the values are quite good. For instance, for A+STU on ballistic missiles, the main peak is around 0.85 . After the application of the utility function, the probability distribution becomes much less optimistic. For instance, for A+STU on ballistic missiles, the main peak is around 0.3 with some kind of plateau between 0.7 and 1. The overall score presented in the gauge is the integral of the product of the blue and the red curves. We note that the red curve (OWA weights) are decreasing, which means that more weights are put on the worst tracked 


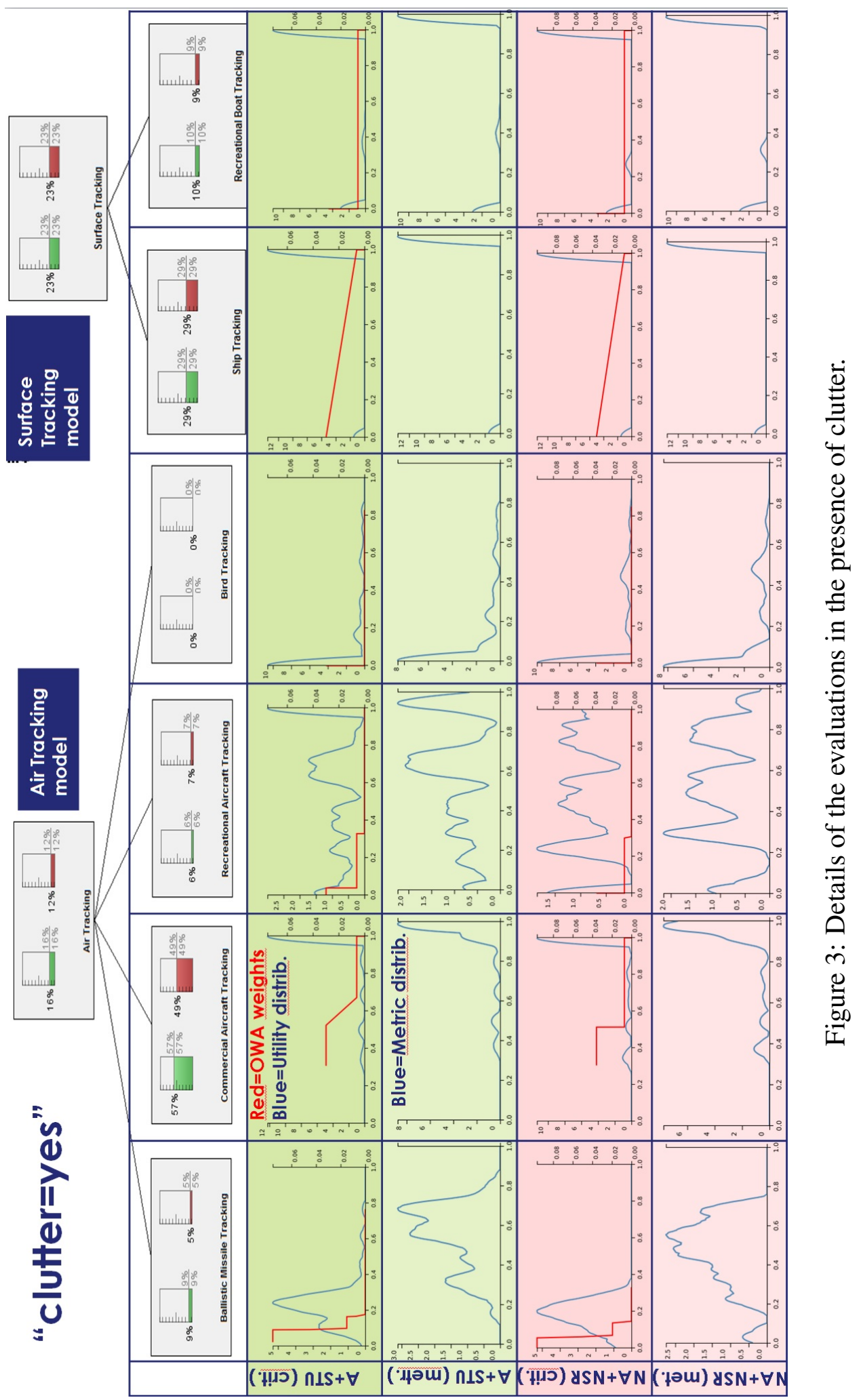

Ch. Labreuche, C. Buron, P. Moo, F. Barbaresco, "Multi-criteria Performance Assessment of Adaptive 
targets. This explains the bad evaluations on the gauges.

Case where there is no clutter There is significant improvement on A+STU compared to NA+NSR, except for Surface tracking for which the results are similar. A+STU gets very good performances on "Commercial Aircraft Tracking", "Recreational Aircraft Tracking" and "Bird Tracking". This is impressive, especially considering the pessimistic OWA weights. On the other hand, there is no major improvement on ballistic missiles tracking. The values of this metric are relative wide spread on the $[0,1]$ range; hence there is not at least $90 \%$ of well-tracked ballistic missiles, which explains the bad evaluation. Overall, A+STU has better performance in all 3 of the main criteria: tracking, surveillance and load balancing. We note that, less tracking beams are needed to track targets for Adaptive RRM (alternative A+STU), which improves load balancing. This extra saved times allows Adaptive RRM to improve the surveillance performance (time frame) over the nonadaptive RMM.

Case where there is some clutter The general comments are similar to the case without clutter. We see that the presence of clutter has very little consequence on surveillance and load balancing, which is a positive point. However, the tracking performances are much lower, especially for "Recreational Aircraft Tracking" and "Bird Tracking". Only "Commercial Aircraft Tracking" maintains a good performance (which is natural as these tracks are easier to track).

\section{Conclusion}

By integrating preferences of the decision maker(s) on his expectations and priorities, MCDA helps connecting the operational perspective on solutions of experts and technical analysis to end users. The main advantages of our approach are to provide transparency on the evaluation process and to show to the user where the figures come from, which is important to gain acceptance and trust by the enduser.

\section{References}

[1] F. Barbaresco, J.C. Deltour, G. Desodt, B.Durand, T. Guenais, and Ch. Labreuche. Intelligent M3R radar time resources management: Advanced cognition, agility \& autonomy capabilities. In International Radar Conference, Bordeaux, France, October 12-16 2009.

[2] F. Barbaresco and R. Wills. Intelligent radar management by advanced beamscheduling algorithms based on time constraints relaxation strategies. In IEEE International Symposium on Phased Array Systems, Boston, Massachusetts, USA, Oct 2003.

[3] Yann Briheche, Frédéric Barbaresco, Fouad Bennis, and Damien Chablat. Optimization of radar search patterns in localized clutter and terrain masking under direction-specific scan update rates constraints. IET Radar Sonar and Navigation, 2018.

[4] Yann Briheche, Frédéric Barbaresco, Fouad Bennis, and Damien Chablat. 
Theoretical complexity of grid cover problems used in radar applications. Journal of Optimization Theory and Applications, 179(3):1086-1106, Dec 2018.

[5] G. Choquet. Theory of capacities. Annales de l'Institut Fourier, 5:131-295, 1953.

[6] Carlos A Bana e Costa, Jean-Marie De Corte, and Jean-Claude Vansnick. MACBETH. International Journal of Information Technology and Decision Making, 11:359-387, 2012.

[7] Carlos A Bana e Costa, Jean-Marie De Corte, and Jean-Claude Vansnick. On the mathematical foundations of macbeth. In Multiple criteria decision analysis, pages 421-463. Springer, 2016.

[8] Vassiliy A Epanechnikov. Nonparametric estimation of a multivariate probability density. Theory of Probability \& Its Applications, 14(1):153-158, 1969.

[9] M. Grabisch. The application of fuzzy integrals in multicriteria decision making. European J. of Operational Research, 89:445-456, 1996.

[10] M. Grabisch and Ch. Labreuche. A decade of application of the Choquet and Sugeno integrals in multi-criteria decision aid. Quarterly Journal of Operations Research (4OR), 6:1-44, 2008.

[11] M. Grabisch and Ch. Labreuche. Fuzzy measures and integrals in MCDA. In M. Ehrgott J. Figueira, S. Greco, editor, Multiple Criteria Decision Analysis -
State of the Art Surveys, pages 553-603. Springer's International Series, 2016.

[12] V. Jeauneau, F. Barbaresco, and T. Guenais. Radar tasks scheduling for a multifunction phased array radar with hard time constraint and priority. In International Radar Conference, RADAR 14, Lille, France, Oct 2014.

[13] Ralph L Keeney and Howard Raiffa. Decisions with multiple objectives: preferences and value trade-offs. Cambridge university press, 1993.

[14] D.H. Krantz, R.D. Luce, P. Suppes, and A. Tversky. Foundations of measurement, volume 1: Additive and Polynomial Representations. Academic Press, 1971.

[15] Christophe Labreuche. Argumentation of the results of a multi-criteria evaluation model in individual and group decision aiding. In Int. Conf. Of the Euro Society for Fuzzy Logic and Technology (EUSFLAT), pages 482-487, Barcelona, Spain, September 7-9 2005.

[16] Christophe Labreuche. A general framework for explaining the results of a multi-attribute preference model. Artificial Intelligence, 175:1410-1448, 2011.

[17] Christophe Labreuche, Frederic Barbaresco, D.Nguyen, T.Guenais, and F. Gosselin. Multi-criteria aggregation for adaptive multifunction radar resource management performances evaluation. In International Radar Symposium (IRS 2017), Prague, Czech Republic, June 2017. 
[18] Christophe Labreuche and Simon Fossier. Explaining multi-criteria decision aiding models with an extended shapley value. In Proceedings of the Twenty-Seventh International Joint Conference on Artificial Intelligence (IJCAI 2018), pages 331-339, Stockholm, Sweden, July 2018.

[19] Christophe Labreuche and Michel Grabisch. The Choquet integral for the aggregation of interval scales in multicriteria decision making. Fuzzy Sets \& Systems, 137:11-26, 2003.

[20] Christophe Labreuche and Fabien Le Huédé. Myriad: a tool suite for MCDA. In Int. Conf. of the Euro Society for Fuzzy Logic and Technology (EUSFLAT), pages 204-209, Barcelona, Spain, September 7-9 2005.

[21] Peter W Moo and Zhen Ding. Adaptive Radar Resource Management. Aca- demic Press, 2015.

[22] Peter W Moo and Zhen Ding. Coordinated radar resource management for networked phased array radars. IET Radar, Sonar \& Navigation, 9(8):10091020, 2015.

[23] Marion Pilté, Silvere Bonnabel, and Frédéric Barbaresco. Fully adaptive update rate for non-linear trackers. IET Radar, Sonar and Navigation, 12:14191428(9), December 2018.

[24] R. R. Yager. On ordered weighted averaging aggregation operators in multicriteria decision making. IEEE Trans. Systems, Man \& Cybern., 18:183-190, 1988.

[25] R. R. Yager. Quantifier guided aggregation using OWA operators. Int. J. of Intelligent Systems, 11:49-73, 1996. 